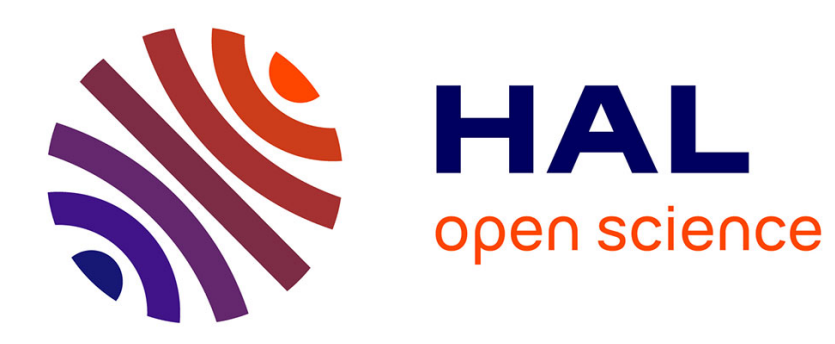

\title{
HYTEC results of the MoMas reactive transport benchmark
}

Vincent Lagneau, Jan van Der Lee

\section{To cite this version:}

Vincent Lagneau, Jan van Der Lee. HYTEC results of the MoMas reactive transport benchmark. Computational Geosciences, 2010, 14, pp.435-449. 10.1007/s10596-009-9159-5 . hal-00505360

\section{HAL Id: hal-00505360 \\ https: / hal-mines-paristech.archives-ouvertes.fr/hal-00505360}

Submitted on 23 Jul 2010

HAL is a multi-disciplinary open access archive for the deposit and dissemination of scientific research documents, whether they are published or not. The documents may come from teaching and research institutions in France or abroad, or from public or private research centers.
L'archive ouverte pluridisciplinaire HAL, est destinée au dépôt et à la diffusion de documents scientifiques de niveau recherche, publiés ou non, émanant des établissements d'enseignement et de recherche français ou étrangers, des laboratoires publics ou privés. 


\title{
HYTEC results of the MoMas reactive transport benchmark
}

\author{
Vincent Lagneau · Jan van der Lee
}

Received: date / Accepted: date

\begin{abstract}
A specific benchmark has been developed by the french research group MoMas in order to improve numerical solution methods applied by reactive transport models, i.e. codes which couple hydrodynamic flow and mass transport in porous media with geochemical reactions. The HYTEC model has been applied to this benchmark exercise and this paper summarizes some of the principal results. HYTEC is a general-purpose code, applied by industrials and research groups to a wide variety of domains, including soil pollution, nuclear waste storage, cement degradation, water purification systems, storage of $\mathrm{CO}_{2}$ and valorization of stabilized wastes. The code has been applied to the benchmark test-cases without any specific modification. Apart from the benchmark imposed output, additional information is provided to highlight the behavior of HYTEC specifically and the simulation results in particular.
\end{abstract}

Keywords reactive transport $\cdot$ HYTEC $\cdot$ benchmark $\cdot$ numerical methods $\cdot$ MoMas

\section{Introduction}

Reactive transport models have proven to be effective tools to help us to understand the behavior of hydrodynamically and chemically reactive systems [14], as demonstrated by the wide variety of applications, in numerous domains and at different scales. However, the equations involved are complex and non-linear, including coupled partial differential equations and algebro-differential systems of equations, so that it is difficult to demonstrate that code simulations are accurate. Several possiblities are open to test the precision and accuracy of the codes. Analytical solutions are the ideal tool, when available [5, 15,21,13]; however, they are not easy to devise and are limited to simplified systems: specific hydrodynamic, simplistic chemistry, weak feedbacks. It is also possible to use laboratory or field

V. Lagneau

Mines ParisTech, 35 rue Saint Honoré, 77305 Fontainebleau Cedex, France

Tel.: +1.64.69.49.36

Fax: +1.64.69.47.13

E-mail: vincent.lagneau@mines-paristech.fr

J. van der Lee

same address 
experiments $[6,12]$. However, experiments do not provide an irrefutable validation models: due to the complexity of the systems and the unavoidable lack of data (especially for heterogeneous systems, with possibly complex relations between mineralogy, porosity, reactive surface area, permeability), some parametrisation of the simulations remains. Finally, code intercomparison can help validate the codes, provided that the specifications of the exercises are weel defined $[7,4]$. In this paper, we detail the solutions obtained by the HYTEC code [19], developed at Mines ParisTech, on a suite of benchmark exercises.

\subsection{The reactive transport benchmark MoMaS}

In 2006, the MoMaS research group proposed a suite of exercises to test reactive transport models $[1-3]^{1}$. The major objective of the benchmark is to provide more efficient numerical methods and mathematical resolution schemes in order to improve reactive transport models, in particular for applications in the domain of deep underground radioactive waste disposal. Contrary to the approach suggested by [5], the benchmark exercise does not focus on an existing analytical solution, but rather on problems with strong coupling and stiffness. The lack of reference solution will cause some problems when the accuracy of the results will be discussed.

The benchmark is composed of three subsequent cases with increasing chemical complexity, named "easy", "medium" and "hard". The systems do not represent real chemical systems: they were devised by [1] to create numerical difficulties for the resolution. For the easy case, the chemistry is composed of 4 basis components $\mathrm{X} 1, \ldots, \mathrm{X} 4$, and a surface site $\mathrm{S}$. The other chemical species can be described as linear composed of these basis components, at equilibrium:

$$
\begin{array}{lll}
\text { aqueous species } & & \\
\mathrm{C} 1 \rightleftharpoons-\mathrm{X} 2 & & K=10^{-12} \\
\mathrm{C} 2 \rightleftharpoons \mathrm{X} 2+\mathrm{X} 3 & K=1 \\
\mathrm{C} 3 \rightleftharpoons-\mathrm{X} 2+\mathrm{X} 4 & K=1 \\
\mathrm{C} 4 \rightleftharpoons-4 \mathrm{X} 2+\mathrm{X} 3+3 \mathrm{X} 4 & K=0.1 \\
\mathrm{C} 5 \rightleftharpoons 4 \mathrm{X} 2+3 \mathrm{X} 3+\mathrm{X} 4 & K=10^{35} \\
\text { surface sites } & & \\
\mathrm{CS} 1 \rightleftharpoons 3 \mathrm{X} 2+\mathrm{X} 3+\mathrm{S} & K=10^{6} \\
\mathrm{CS} 2 \rightleftharpoons-3 \mathrm{X} 2+\mathrm{X} 4+2 \mathrm{~S} & K=10^{-1}
\end{array}
$$

Initial and boundary conditions are defined by the total concentration of each basis component Tab. 1

For the medium case, additional reactions are taken into account:

$$
\begin{aligned}
& \mathrm{C} 6 \rightleftharpoons 10 \mathrm{X} 2+3 \mathrm{X} 3 \quad K=10^{32} \\
& \mathrm{C} 7 \rightleftharpoons-8 \mathrm{X} 2+2 \mathrm{X} 4 \quad K=10^{-4} \\
& \mathrm{Cc} \rightleftharpoons-3 \mathrm{X} 2+\mathrm{X} 4 \quad K_{c}=0.2
\end{aligned}
$$

where $\mathrm{Cc}$ is a non-mobile species; its reaction is kinetically controlled following:

$$
\frac{\mathrm{dCc}}{\mathrm{d} t}=k\left[0.2 \frac{\mathrm{C} 3^{3}}{\mathrm{X} 4^{2}}-1\right] \text { where }\left\{\begin{array}{l}
k=10^{-2} \text { if } 0.2 \frac{\mathrm{C} 3^{3}}{\mathrm{X} 4^{2}} \geq 1 \\
k=10 \text { otherwise }
\end{array}\right.
$$

1 GdR-MoMaS: http://www.gdrmomas.org, definition of the exercise: http://www.gdrmomas . org/Ex_qualif/Geochimie/Documents/Benchmark-MoMAS.pdf 


\begin{tabular}{lccccc}
\hline Total & T1 & T2 & T3 & T4 & TS \\
\hline Easy test case & & & & & \\
medium A & 0 & -2 & 0 & 2 & 1 \\
medium B & 0 & -2 & 0 & 2 & 10 \\
injection & 0.3 & 0.3 & 0.3 & 0 & \\
leaching & 0 & -2 & 0 & 2 & \\
\hline Medium test case & & & & \\
medium A & 0 & -3 & 0 & 1 & 1 \\
medium B & 0 & -9 & 0 & 3 & 10 \\
injection & 0.3 & 0.3 & 0.3 & 0 & \\
leaching & 0 & -3 & 0 & 1.5 & \\
\hline
\end{tabular}

Table 1 Chemical composition of the initial and boundary conditions for the easy and medium test cases.

\begin{tabular}{lcc}
\hline configuration & medium A & medium B \\
\hline porosity & 0.25 & 0.5 \\
permeability & $10^{-2}$ & $10^{-5}$ \\
darcy velocity & \multicolumn{2}{c}{$5.5 \times 10^{-3}$} \\
advective case & \multicolumn{2}{c}{} \\
dispersivity $\alpha_{L}$ & $10^{-2}$ & $6 \times 10^{-2}$ \\
dispersivity $\alpha_{T}$ & $10^{-3}$ & $6 \times 10^{-3}$ \\
dispersive case & & \\
dispersivity $\alpha_{L}$ & 10 & 60 \\
dispersivity $\alpha_{T}$ & 1 & 6 \\
\hline
\end{tabular}

Table 2 Hydrodynamic parameters used for the benchmark MoMaS; the advective and dispersive configurations are detailed. All parameters are given adimensionally, in a relevant system of physical units.

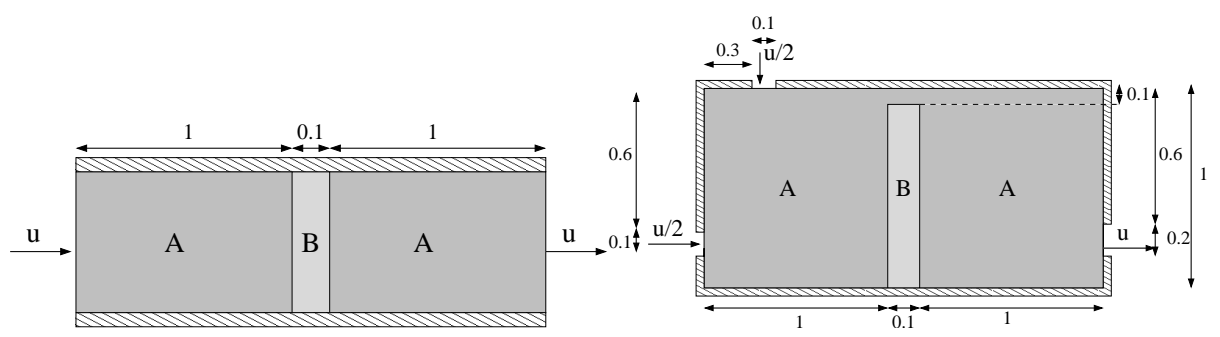

Fig. 1 Geometry of the 1D and 2D cases.

The transport processes are advective/dispersive. However, each chemical case is derived under two dispersivity specifications, which allows to test the models with respect to numerical stiffness: mainly advective or mainly dispersive situations (Tab. 2). Eventually, each case is treated in a 1D and 2D geometry (Fig. 1). The exercise also tests the stiffness introduced by a modification of the boundary conditions: the injected solution changes (from "injection" to "leaching", Tab. 1) at time 5000 for a total simulation time of 6000 .

\subsection{Benchmarking exercise}

The benchmark exercises test the numerical behavior of reactive transport codes. The main features needed to solve the problems are: 
- a chemical solver for aqueous complexes and surface sites at equilibrium (easy case), and kinetically limited mineral (medium case);

- an advective/dispersive transport solver; anisotropic transport should be available for the $2 \mathrm{D}$ variation;

- a 2D flow solver for the 2D variation.

The kinetic law is basic [11] and widely available in general chemical solver. For the hard case, an additional unstable basis component is used. The dissociation law for species X5

$$
\mathrm{X} 5 \rightarrow 2 \mathrm{X} 2+\mathrm{X} 3
$$

does not follow a usual disintegration law: the reaction rate depends on the concentration of species X5 and a secondary mineral. The resolution of this unusual problem requires a specific development to implement this dissociation law. Part of the originality of the HYTEC contribution to the benchmark is that it uses the standard version of HYTEC; for this reason, the resolution of the hard case was not attempted.

Several research institutes worked on the benchmark, using a variety of numerical codes, and the results were compared during an international workshop in Strasbourg in january 2009, and collectively presented and compared (this issue). The complete set of results using HYTEC at Mines ParisTech for the easy and medium, advective and dispersive, 1D and 2D test-cases are available in two technical reports $[9,16]$. This paper mainly aims at providing a comprehensive evaluation of the numerical and physico-chemical results for the base-case of the study. This part focusses on the one-dimensional geometry of the easy case. HYTEC is described with a focus on the numerical methods relevant to the test-case; the modeling results are given according to the standard format as imposed by the benchmark organizers. And finally, a more in-detail discussion is provided in order to explain the specific results and numerical behavior of HYTEC.

\subsection{The reactive transport code HYTEC}

HYTEC is a model which allows to solve for the reactive transport system. It therefore couples hydrodynamic flow and multi-component transport with biogeochemical processes [19]. HYTEC is developed within the framework of the Reactive Transport Consortium ${ }^{2}$, a research group which includes industrial and academic partners. This structure not only allows to develop HYTEC on a long-term basis - it also imposes a wide variety of application domains, from small scales material science (e.g. corrosion of steal, waste-glass dissolution, degradation of cement) to large-scale geological problems (e.g. storage of $\mathrm{CO} 2$, radioactive waste disposal). HYTEC therefore aims to be a versatile, configurable and operational tool, applicable to any reactive transport problem. Within this context, it is useful to mention that HYTEC is a massively parallel code and can be run on large multi-processor computers.

In its current version, HYTEC deals with stationary and transient flow and multi-component transport of water in saturated conditions. Unsaturated conditions are accurately modeled for transient flow and transport only. Heat transport is accounted for, coupled with flow (heat advection, feedback on water viscosity). The hydrodynamic system is solved by R2D2, a model specifically developed for use with HYTEC.

All chemical or biogeochemical reactions are solved by the speciation code CHESS [17], linked with HYTEC. CHESS is a versatile geochemical speciation code, and consequently allows for the simulation of aqueous speciation, precipitation and dissolution of

\footnotetext{
2 Pôle Géochimie-Transport or PGT: http://pgt.geosciences .ensmp.fr.
} 
solid (mineral) phases, interface reactions (based on a choice of surface complexation and ion exchange models), temperature dependencies, radioactive decay with multi-ancestor and multi-descendant filiation, to cite a few of the possibilities. The model is unlimited with respect to the number of species taken into account. The species and reactions are defined by extensive thermodynamic databases, e.g. the one provided by the Common Thermodynamic Database Project ${ }^{3}$ All reactions can be modeled using the local equilibrium assumption, full kinetic control or a mixture of both. Microbial reactions are accounted for by specific kinetic reaction laws, including inhibiting or catalyzing (Monod-like) reaction terms. Coupled with HYTEC, CHESS uses an improved Newton-Raphson scheme to solve the set of non-linear algebraic equations. The Jacobian matrix, required by the method, is solved analytically even if kinetics are involved. This improves the convergence speed significantly and greatly contributes to the efficiency of HYTEC.

\section{Numerical methods}

The hydrodynamic module R2D2 [8] simulates flow coupled with heat and multicomponent solute transport. The code uses a finite volume scheme based on a Voronoi (nearestneighbour) grid in one, two or three dimensions. The discretization scheme can be chosen between centered (default for the dispersion) and upstream (default for the advection). The time discretization is a one step scheme and can be set from fully explicit to implicit, with a centered (Crank-Nicholson) approach by default. For an explicit scheme in pure transport, the time step must follow the Courant-Friedrichs-Levy (CFL) criterion:

$$
\delta t<\frac{\omega \delta x / 2}{U(1+4 \alpha / \delta x)}
$$

for a $1 \mathrm{D}$ uniform grid (with $\omega$ the porosity, $U$ the local Darcy velocity, and $\alpha$ the dispersivity). It derives from this relation that the higher the dispersivity, the smaller the admissible time step. An approximation of the optimal (i.e. largest possible) time step is calculated at each time step to account for possible changes in the velocity field. The calculated value is an approximation by default only, since it is calculated based on global parameters of each cell (i.e. volume and surface area) and not the actual shape of the cell, which can be limiting sometimes. To correct for this approximation, to account for larger (but not assessable) CFL criterion in presence of chemical source terms, and to offer the possibility of using larger time steps in semi-implicit or implicit schemes HYTEC provides a multiplier, $f_{c}$, of the time-step based on the numerical CFL criterion. The value of $f_{c}$ is set to 0.5 by default. The effect of different values for $f_{c}$ on the overall CPU needed to run the test-case is discussed within the context of the test-case results.

Coupling of transport and chemistry in HYTEC is based on the sequential iterative approach $[20,19]$. Using the basis component formalism to describe the chemical system, the transport equation can be written as follow:

$$
\frac{\partial \omega \tilde{c}_{i}}{\partial t}=\mathscr{L}\left(\tilde{c}_{i}\right)-\frac{\partial \omega \bar{c}_{i}}{\partial t}
$$

where $\tilde{c}_{i}$ is the mobile fraction of the total concentration of component $i$ and $\bar{c}_{i}$ is the immobile fraction, such that the total concentration yields $c_{i}=\tilde{c}_{i}+\bar{c}_{i} \cdot \mathscr{L}$ is the transport operator $\mathscr{L}(c)=\operatorname{div}\left(D_{e} \underline{\operatorname{grad}} c-c \underline{U}\right)$. The most right-hand term represents the chemical source/sink

\footnotetext{
3 CTDP:http://www.ctdp.org.
} 
term: although they are not explicitly written Eq. 2, the chemical reactions are responsible for the balance of fixed components. Indeed, due to the chemical reactions, the local composition of the system changes, with the possible transformation of mobile species into fixed species, therefore modifying the balance of basis components between the mobile and fixed fraction. This equation can be split, using the discrete unknown series $\left(\omega^{t, m}, \tilde{c}^{t, m}, \bar{c}^{t, m}\right)$. Supposing time step $t$ determined and iteration $(t+\delta t, 2 m)$ completed, we can write a transport iteration step $(t+\delta t, 2 m+1)$ solving the transport globally for each species $i$ :

$$
\begin{aligned}
\forall i, \frac{\omega^{t+\delta t, 2 m+1} \tilde{c}_{i}^{t+\delta t, 2 m+1}-\omega^{t} \tilde{c}_{i}^{t}}{\delta t}= & \alpha \mathscr{L}\left(c_{i}^{t+\delta t, 2 m+1}\right)+(1-\alpha) \mathscr{L}\left(c_{i}^{t}\right) \ldots \\
& \cdots+\frac{\omega^{t+\delta t, 2 m} \bar{c}_{i}^{t+\delta t, 2 m}-\omega^{t} \bar{c}_{i}^{t}}{\delta t}
\end{aligned}
$$

where the chemical source term is calculated relative to iteration $(t+\delta t, 2 m)$. A new chemical iteration step $(t+\delta t, 2 m+2)$ is then performed, solving the speciation globally for all species at each node $x$ :

$$
\forall x,\left(\omega_{x}^{t+\delta t, 2 m+2}, \tilde{c}_{x}^{t+\delta t, 2 m+2}, \bar{c}_{x}^{t+\delta t, 2 m+2}\right)=\chi\left(\tilde{c}_{x}^{t}, \bar{c}_{x}^{t}, \frac{\omega_{x}^{t+\delta t, 2 m+1} \tilde{c}_{x}^{t+\delta t, 2 m+1}-\omega_{x}^{t} \tilde{c}_{x}^{t}}{\delta t}\right)
$$

where the transport mass variation is calculated relative to the previous iteration $(t+\delta t, 2 m+$ 1 ). All chemistry is accounted for by the reaction operator $\chi$, which, using the total concentration $\left(\tilde{c}_{x}^{t}+\bar{c}_{x}^{t}\right)$, provides an appropriate value for the retained amount of species, $\bar{c}_{x}^{t}$. The HYTEC model calls the solution modules provided by the chemical speciation code CHESS, which provides solutions for thermodynamic equilibrium, kinetic or mixed systems.

The guess $\left(\omega^{t+\delta t, 0}, \tilde{c}^{t+\delta t, 0}, \bar{c}^{t+\delta t, 0}\right)$ is carefully calculated at each new time step on a two-time step basis, in an attempt to minimize the number of coupling iterations needed to reach a reasonable approximation of the limit $\left(\omega^{t+\delta t, \infty}, \tilde{c}^{t+\delta t, \infty}, \bar{c}^{t+\delta t, \infty}\right)=\left(\omega^{t+\delta t}, \tilde{c}^{t+\delta t}\right.$, $\bar{c}^{t+\delta t}$ ). The initial guess for the chemical source term (variation of the fixed quantities) is based on a prolongation at each node of the fixed totals (possibly corrected for the porosity evolution):

$$
\forall i\left\{\begin{aligned}
\frac{\omega_{i}^{t+\delta t, 0}-\omega_{i}^{t}}{\delta t} & =\frac{\omega_{i}^{t}-\omega_{i}^{t-\delta t^{\prime}}}{\delta t^{\prime}} \\
\frac{\omega_{i}^{t+\delta t, 0} \bar{c}_{i}^{t+\delta t, 0}-\omega_{i}^{t} \bar{c}_{i}^{t}}{\delta t} & =\frac{\omega_{i}^{t} \bar{c}_{i}^{t}-\omega_{i}^{t-\delta t^{\prime}} \bar{c}_{i}^{t-\delta t^{\prime}}}{\delta t^{\prime}}
\end{aligned}\right.
$$

It has been showed that this procedure allows to reach convergence faster and to generally reduce stiffness [10].

Convergence is tested after each chemical iteration step for all nodes, with a criterion on the evolution between the iterations of the immobile fractions for each component. The iterative loop includes the porosity update (as can be seen in equations 3 and 4), which allows for dealing with the feedback of chemistry on transport (option not useful for the benchmark). Update of flow and heat equations is not included in the iterative loop: their characteristic time of evolution is usually far less than for reactive transport so that an explicit coupling seems to be a correct approximation for most practical purposes. However, this statement might not hold for specific applications, and a more robust coupling would be then required: $e . g$. near-field of non-isothermal fluid injections (geothermal power generation, $\mathrm{CO}_{2}$ injection), fast moving water-saturation fronts (multiphase flow).

HYTEC automatically optimizes its time step during the runs: the time step increases (decreases) if the number of iterations needed for coupling is less (greater) than a user 
defined value ( 20 by default) within certain limits (total duration and sampling, CourantFriedrichs-Levy criterion if applicable). This allows to decrease the time step when the system becomes harder to solve and to increase the time steps whenever possible, thus decreasing the CPU required to run the case. Furthermore, when coupling is really hard, another procedure is undertaken: the resolution goes back one time step then resumes with a slower time step (-30\% by default) before continuing.

\section{Benchmark results for the "easy case" using HYTEC}

The 1D easy test-case has been solved using HYTEC version 3.6 release 3, the current standard version of HYTEC. The code has been applied as such, no specific modifications have been applied to run faster or to improve convergence. Some essential numerical parameters for the test-case are:

- regular 1D grid, with a sensitivity analysis on the total number of nodes;

- transport scheme: centered in space (no numerical dispersion), one-step semi-implicit in time (Crank-Nicholson);

- precision of the resolution of the transport equations: $10^{-8}$;

- precision of the resolution of the chemical equations (Newton-Raphson): $10^{-8}$;

- precision of coupling between chemistry and transport: $10^{-5}$;

- variable time step, with number of iterations threshold (i.e. "acceptable" coupling stiffness): 20.

The chemical and physical parameters for the simulation are not given in this paper as they are thouroughly described in the benchmark specification [1].

The simulation results are given in figures 2 to 7 for the advective case, figures 8 to 12 for the dispersive case, following the benchmark presentation guideline. The simulations were performed on a regular 1D grid; several grid sizes were chosen to test the effect of the discretization: 105, 126, 210, 420 and 1050 nodes for the total length 2.1 (as shown in the graphs). The transport is solved using a centered scheme, i.e. without numerical dispersion; however, note that the oscillatory pattern observed Fig. 2 is not due to the transport scheme: it was checked that the convergence criterion was respected $(d x \leq 2 \times \alpha$, i.e. 105 nodes or more for the advective case), it is also verified that the non-reactive component $\mathrm{X} 1$ does not display oscillations. Likewise, tests using the upwind scheme yield the same results, providing that the numerical dispersion due to this choice of resolution scheme is deducted from the physical dispersion defined by the exercise specifications. An in-depth explanation on the reason of the oscillations is given in the discussion section 4.

The effect of the time step has also been tested. The effect is limited in terms of simulation results, so that an increased Courant multiplicator $\left(f_{c}=10\right)$ has been used for the advective simulations. However, it can be seen that the gain in total CPU for a simulation reaches a plateau around $f_{c}=20$. Indeed, since the system is non-linear, an increase in the admissible time step does not automatically decrease the effective CPU: the overall effect of an increase in the time step can be obliterated by an larger increase in the required number of iterations.

For the dispersive simulations a $f_{c}=20$ was chosen. It has been verified that this value is near the optimal balance between large time steps and not too stiff coupling: in this particular case, the number of iterations is around 8 for a time step limited by $f_{c}=20$, and 20 iterations for $f_{c}=40$ : no benefit is gained by the increase in admissible time step. Moreover, $f_{c}>40$ 


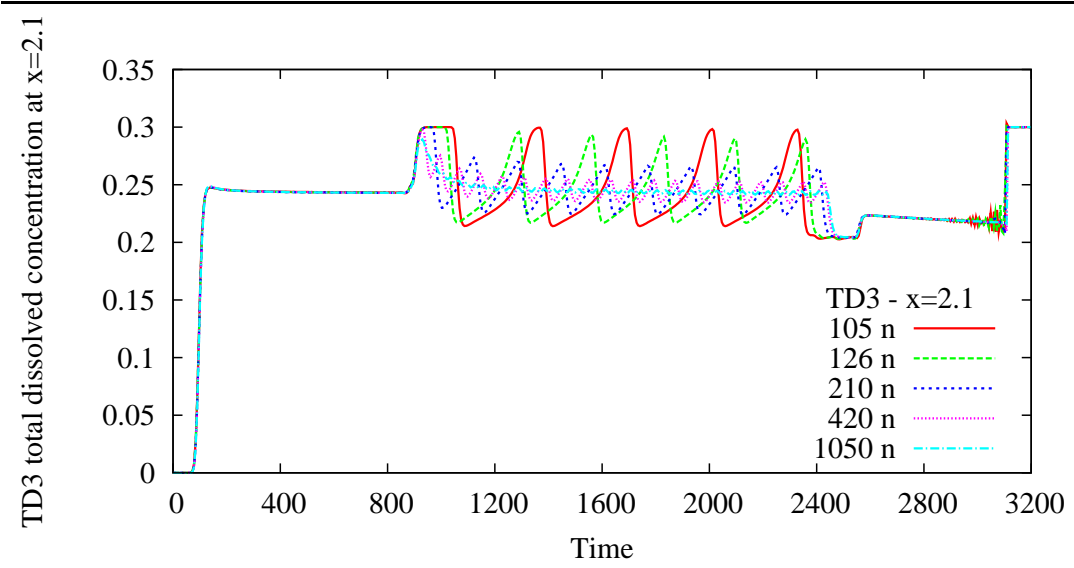

Fig. 2 Elution curve for the advective easy 1D case for total dissolved TD3 at $x=2.1$

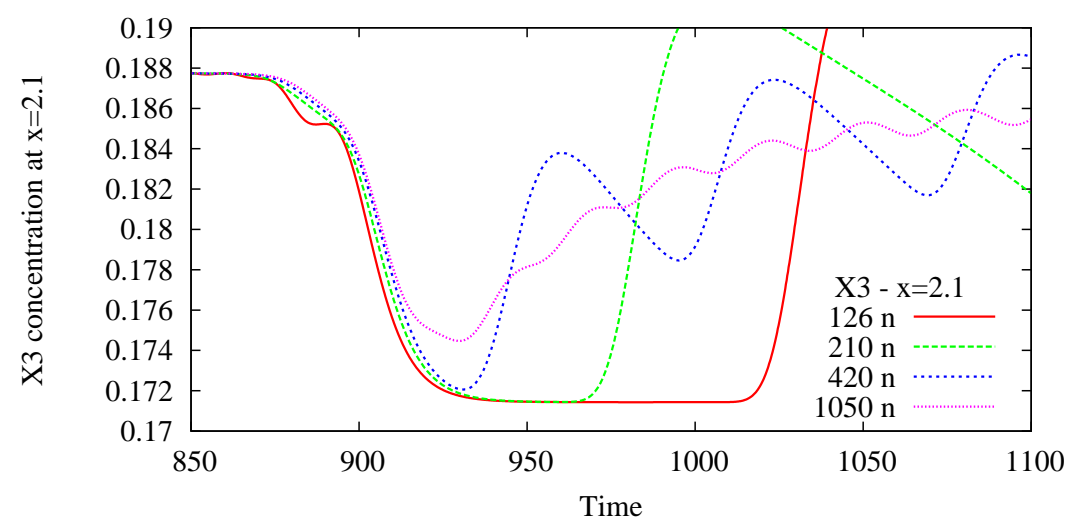

Fig. 3 Elution curve for the advective easy 1D case for concentration of species X3 at $x=2.1$

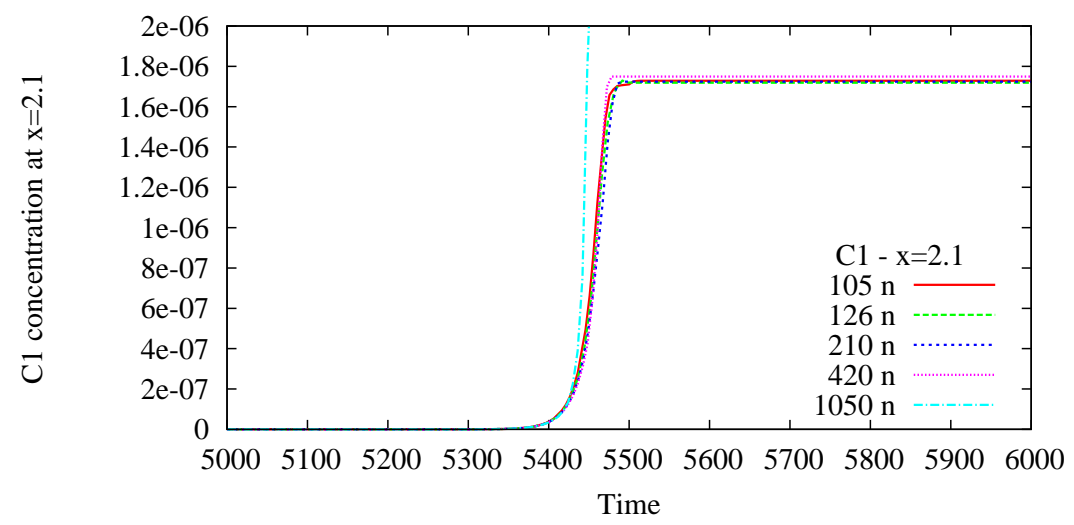

Fig. 4 Elution curve for the advective easy 1D case for concentration of species C1 at $x=2.1$ 


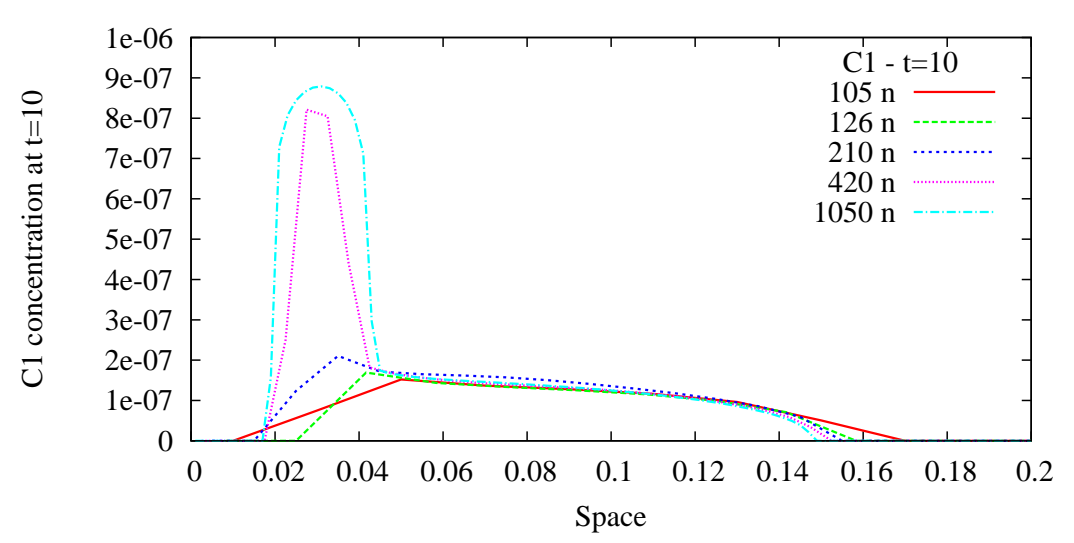

Fig. 5 Concentration profile for the advective easy $1 \mathrm{D}$ case of species $\mathrm{C} 1$ at $t=10$

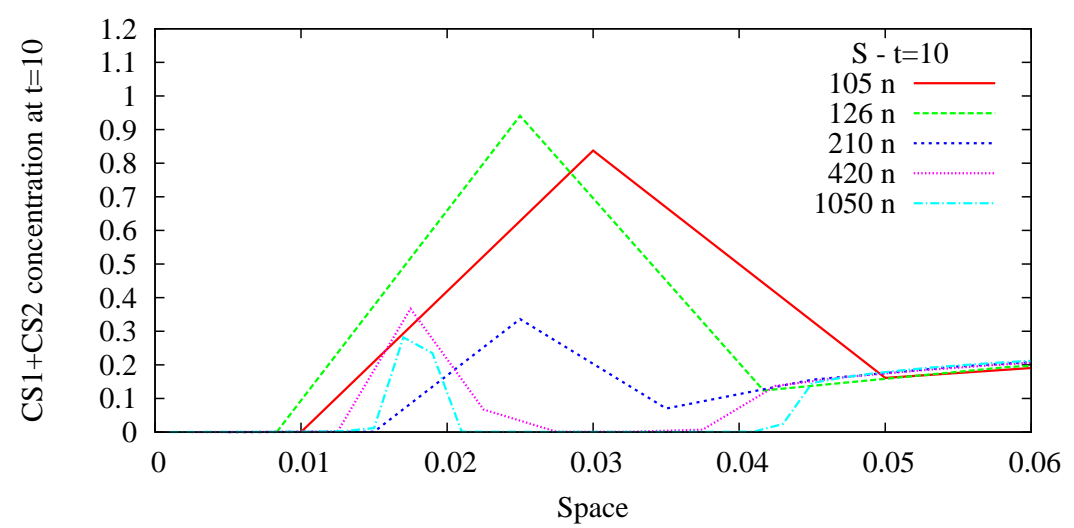

Fig. 6 Concentration profile for the advective easy 1D case of the fixed component $\mathrm{S}$ at $t=10$

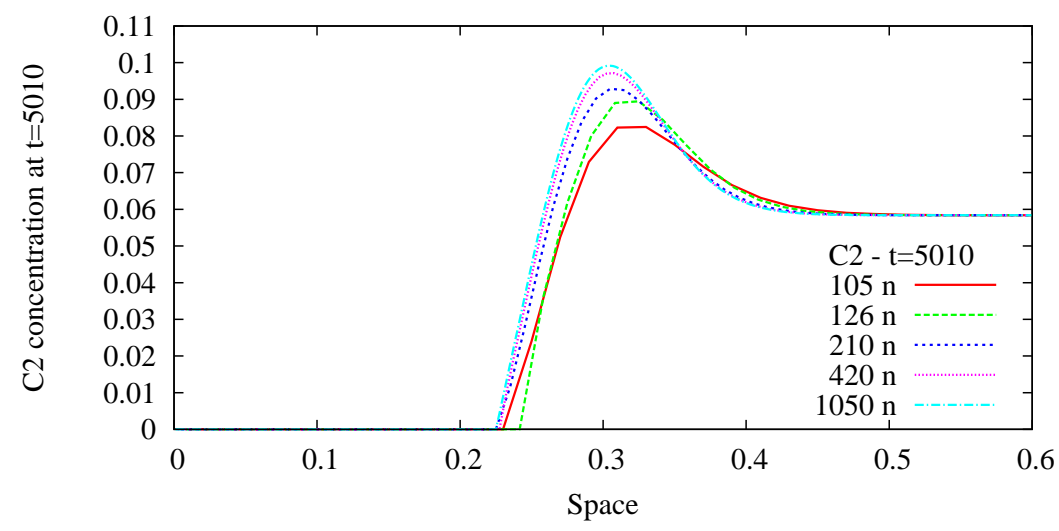

Fig. 7 Concentration profile for the advective easy 1D case of species C2 at $t=5010$ 


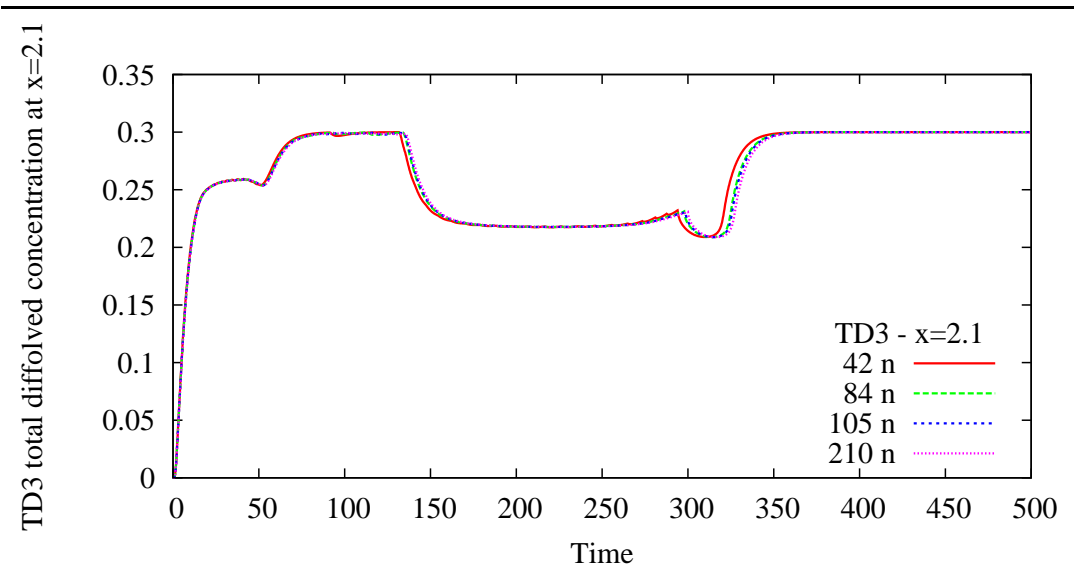

Fig. 8 Elution curve for the dispersive easy 1D case for total dissolved TD3 at $x=2.1$

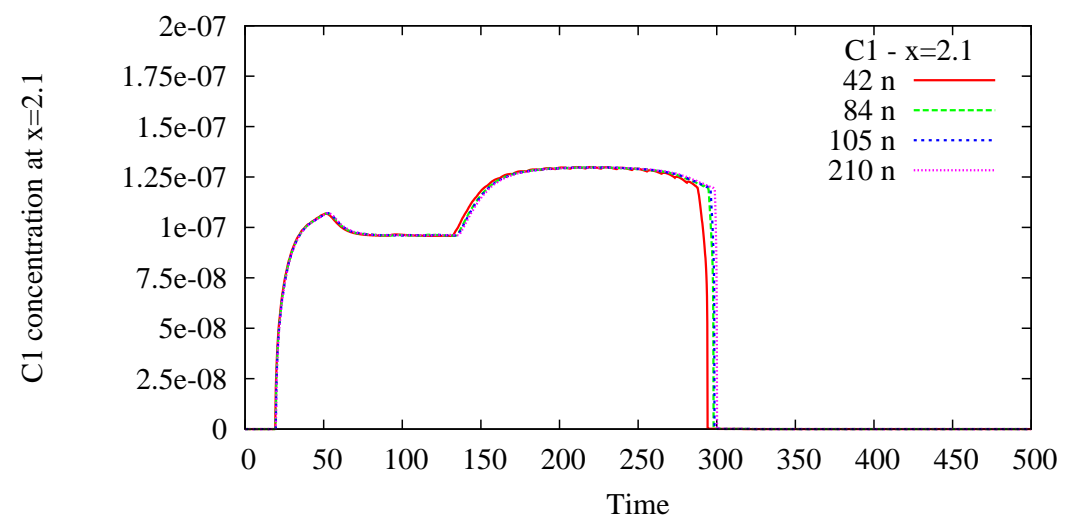

Fig. 9 Elution curve for the dispersive easy 1D case for total species C1 at $x=2.1$

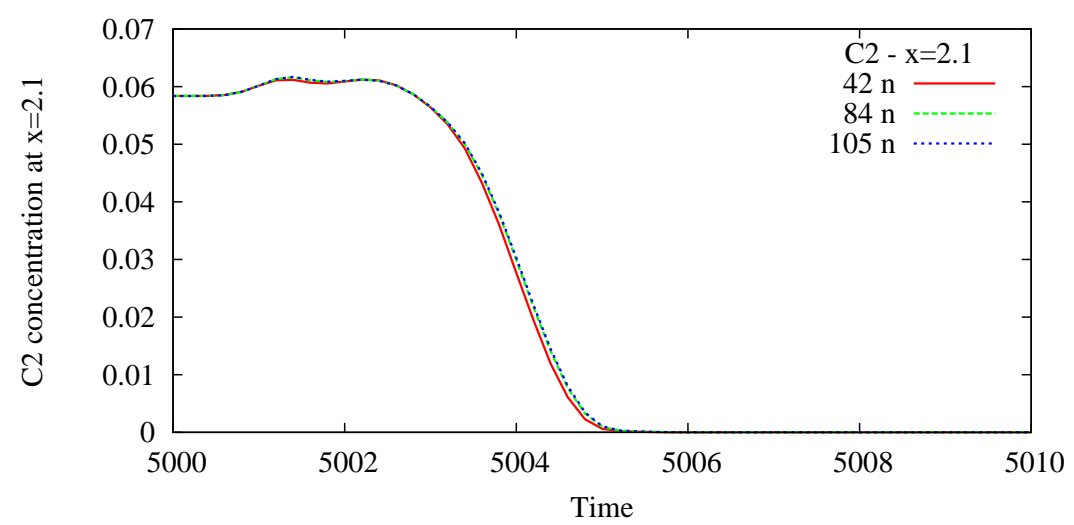

Fig. 10 Elution curve for the dispersive easy 1D case for total species C2 at $x=2.1$ 


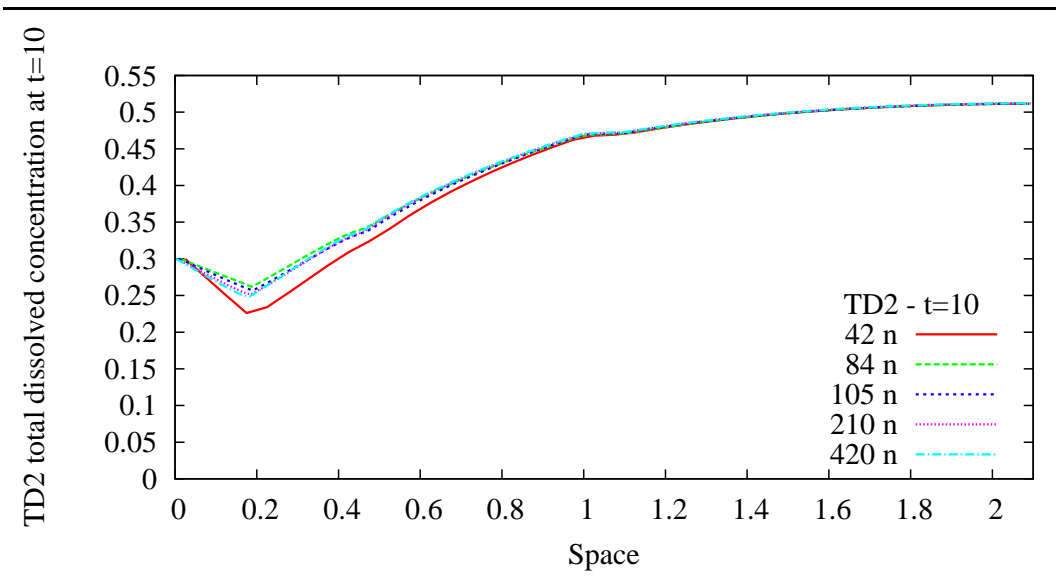

Fig. 11 Concentration profile for the dispersive easy 1D case of the total dissolved TD2 at $t=10$

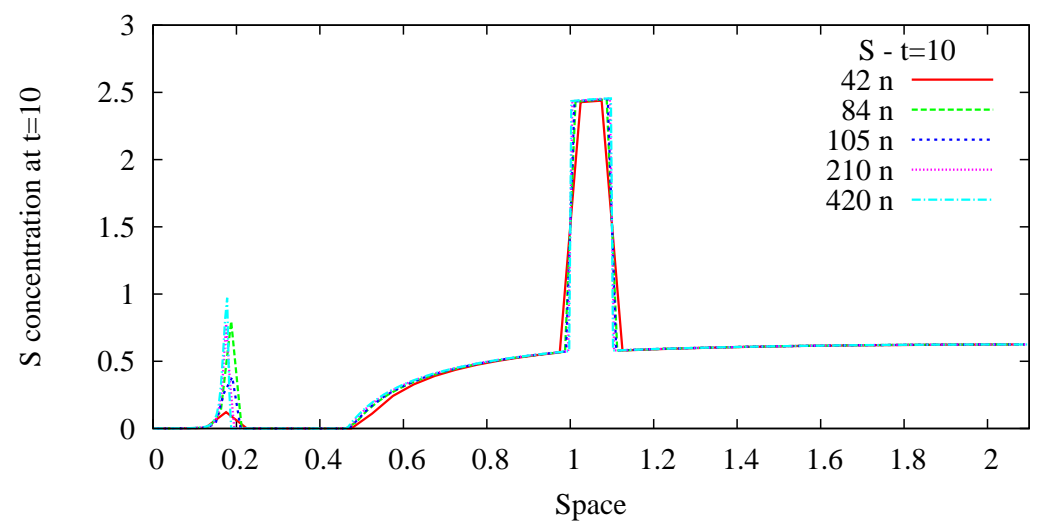

Fig. 12 Concentration profile for the dispersive easy 1D case of the fixed component $\mathrm{S}$ at $t=10$

would result in an effective limitation of the time step via the target number of iterations of coupling in a time step (maximum 20 by default in HYTEC).

The computation times are given in the normalized format in table 3 , in function of the number of nodes and the Courant multiplier. It is useful to mention that HYTEC is massively parallelized: hydrodynamics and chemistry are launched on different processors and the resolution of chemical reactions for all nodes can be distributed on an arbitrary number of processors. Nevertheless, this option has not been used for the current study: chemistry was not parallelized, and chemistry and transport are treated sequentially so that no improvement is made by the parallelization; on the contrary, additional CPU time is spent for message passing.

\section{Discussion on the "easy case" results}

One of the difficulties observed during the advective test-case is the sequence of exchange between fixed species CS2 and CS1. Water containing X2 and X3 (but not X4) is injected at the inlet and creates a disequilibrium with respect to the initially present sorbed species 
Table 3 Normalized computation time using HYTEC. CPU times are given for several values of the Courant factor (see text) and several grid sizes.

\begin{tabular}{|c|c|c|c|c|c|c|}
\hline & \multicolumn{5}{|c|}{ advective case } & \multirow{2}{*}{$\begin{array}{c}\text { dispersive case } \\
f_{c}=20\end{array}$} \\
\hline & $f_{c}=0.99$ & $f_{c}=5$ & $f_{c}=10$ & $f_{c}=20$ & $f_{c}=50$ & \\
\hline 42 nodes & & & 1.02 & & & 355.3 \\
\hline 84 nodes & & & & & & 909.3 \\
\hline 105 nodes & & & 8.73 & & & 1349.0 \\
\hline 210 nodes & 237.43 & 60.39 & 47.26 & 42.2 & 41.57 & \\
\hline 420 nodes & & & 294.24 & & & \\
\hline 1050 nodes & & & 3302.99 & & & \\
\hline
\end{tabular}

Table 4 Chemical speciation in initial zones A and B and for the boundary conditions.

\begin{tabular}{lcccc}
\hline & zone A & zone B & injection & leaching \\
\hline species & & & & \\
X1 & - & - & 0.3 & - \\
X2 & 0.25972 & 1.5116 & 0.24162 & $5.7735 \mathrm{e}-07$ \\
X3 & - & - & 0.24162 & - \\
X4 & 0.34954 & 0.57561 & - & $1.1547 \mathrm{e}-06$ \\
C1 & $3.8503 \mathrm{e}-12$ & $6.6157 \mathrm{e}-13$ & $4.1387 \mathrm{e}-12$ & $1.7321 \mathrm{e}-06$ \\
C2 & - & - & 0.05838 & - \\
C3 & 1.3458 & 0.38081 & - & 2 \\
C4 & - & - & - & - \\
C5 & - & - & - & - \\
\hline sites & & & & \\
TS & 0.39074 & 7.9128 & & \\
CS2 & 0.30463 & 1.0436 & & \\
CS1 & - & - & & \\
\hline total & & - & & \\
TD1 & - & -2 & \\
TD2 & -2 & - & & \\
TD3 & - & 2 & & \\
TD4 & 2 & &
\end{tabular}

CS2 (table 4). The detailed reaction path for a single node shows first the desorption of CS2 which leads to the formation of C5. Then, when all CS2 has been released a second reaction takes place leading to sorption of CS1 due to the arrival of a fresh-water solution and part of a remaining mobile species C5 (figure 13). During the reactive transport process, part of the mobile species C5 migrates downwards in the column, where the dispersion effect smoothes the variations in mobile species profiles.

Due to the assumption of thermodynamic equilibrium, the sorption process takes place in a single node where the reaction front is located at any given time. Accordingly, each moment of CS2 desorption inside a cell leads to a peak in C5, which then migrates downwards, forming oscillations for the C5 profile (and associated species). If this statement is correct, the oscillatory pattern should be a function of the spatial discretization. Indeed, their amplitudes are inversely proportional to the cell size as illustrated by figure 14. Accordingly, it seems to us that these oscillations are a direct consequence of the discretization of the problem and not of the resolution schemes themselves (numerical methods in transport and/or coupling). 

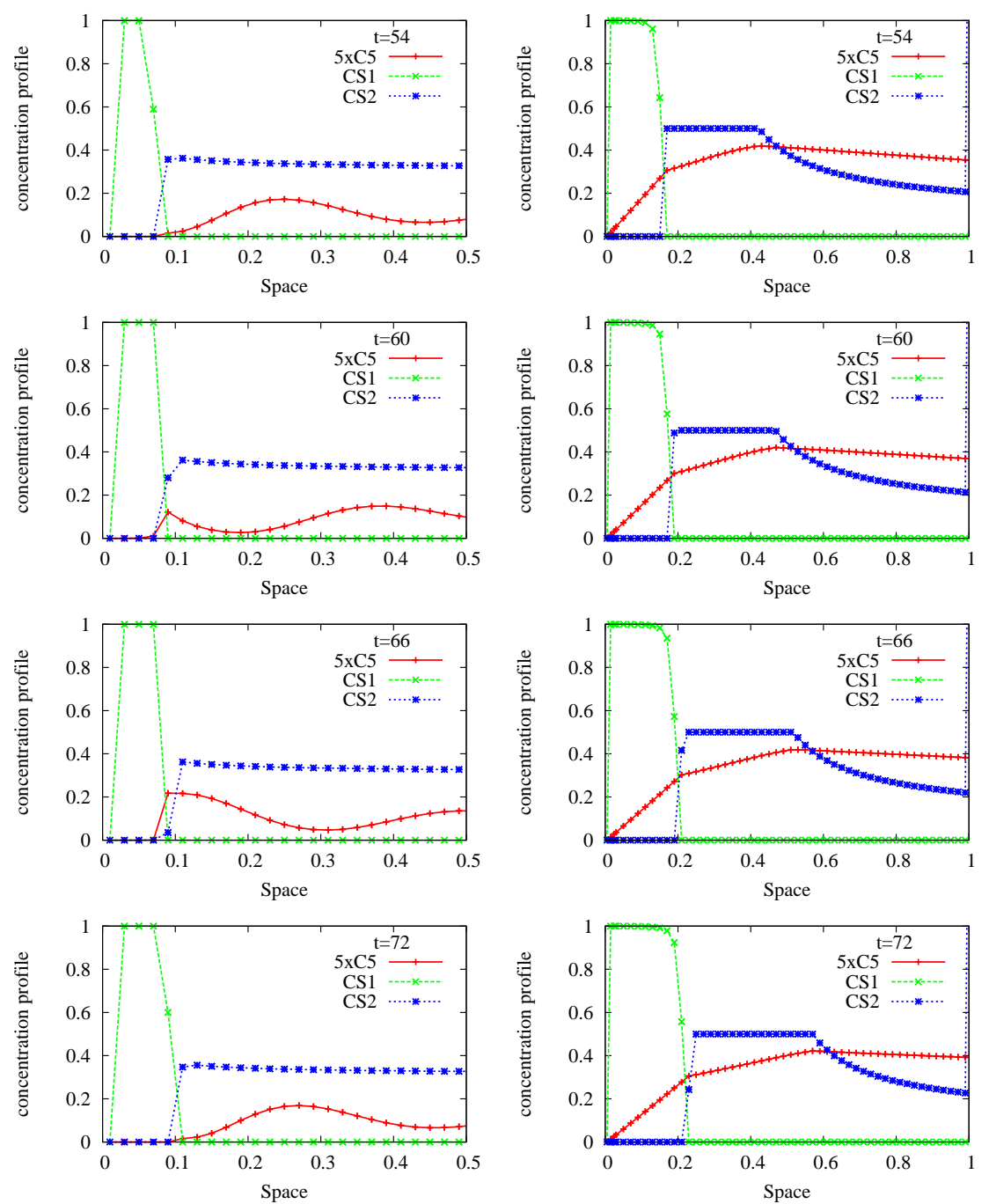

Fig. 13 Concentration profiles for species C5, CS1 and CS2, 105 nodes grid, at several times: $t=54$ and 72 , CS1 desorption with low local C5 concentration, $t=60$ and 66, CS2 sorption creation of a peak of C5. Left: advective case, Right: dispersive case.

The source of oscillation can also be observed in the dispersive case: sequential CS2 to C5 transformation followed by C5 to CS1 formation. However, in this case, the dominant dispersive effect rapidly smoothes the oscillations, so that the $\mathrm{C} 5$ profile remains quasistationary (figure 13). Hence, the effect of the discretization size is much lower for the dispersive case.

In this case, the numerical difficulty comes from a lower Courant number (due to the much larger dispersivity, see eq. 1). Furthermore, the added dispersive component of transport brings in more reactants into the system from the boundary, hence reactions rates are higher than in the advective case. The result is that coupling is harder as can be seen in 

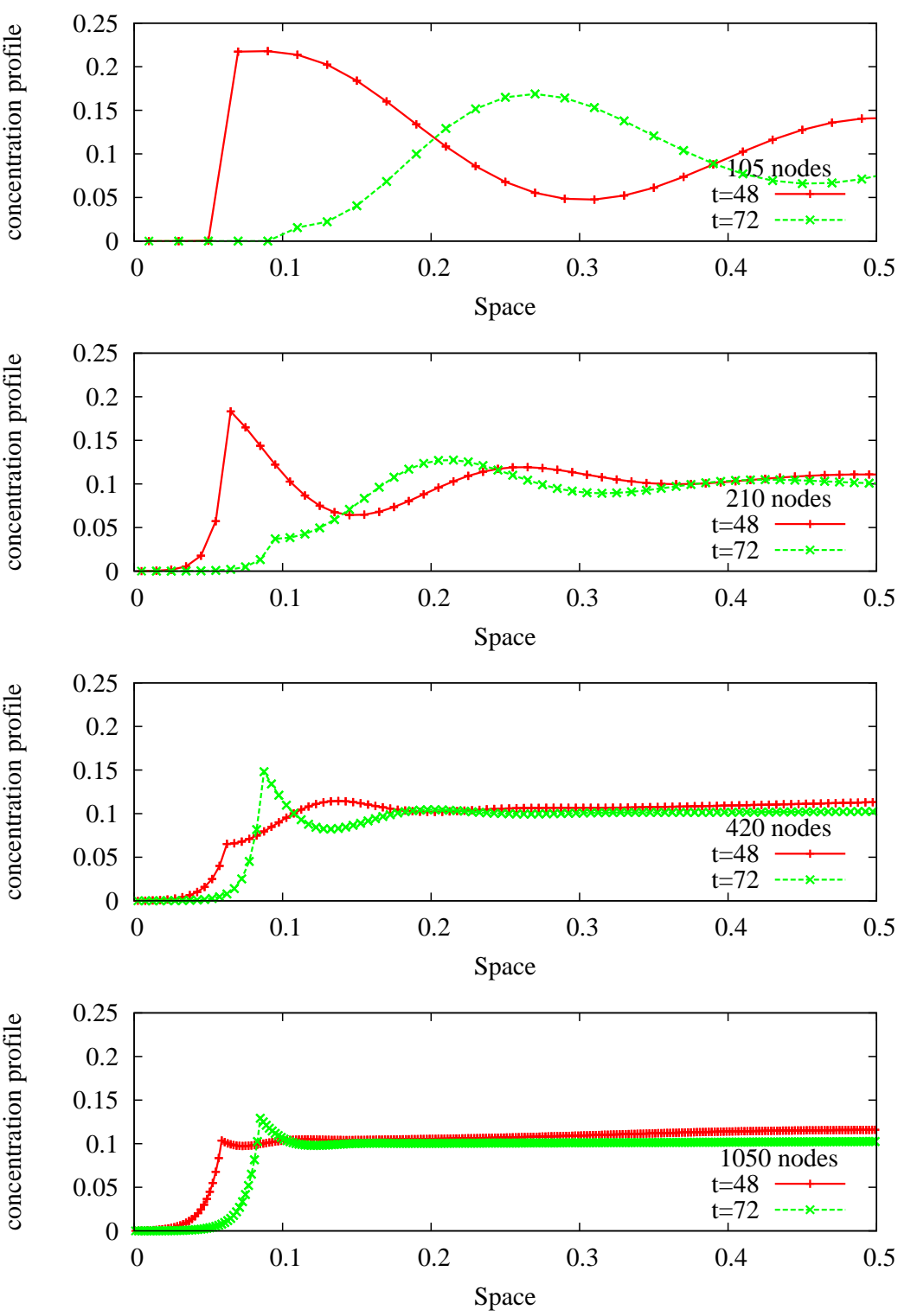

Fig. 14 Concentration profiles for species C5, at $t=48$ and 72, for several grid size.

figure 15 (top), the resolution requiring much more cumulative iterations to reach the same time.

Finally, in both cases, as long as the CS2/CS1 front does not change nodes, the system remains quasi-stationary and the solution is obtained with only few iterations, also because of a fairly accurate guess at the beginning of each time step and low time steps due to the Courant criterion. However, when the front changes nodes, the local chemistry must rearrange, so that guesses become poor, which leads to more iterations. It can be seen that the 

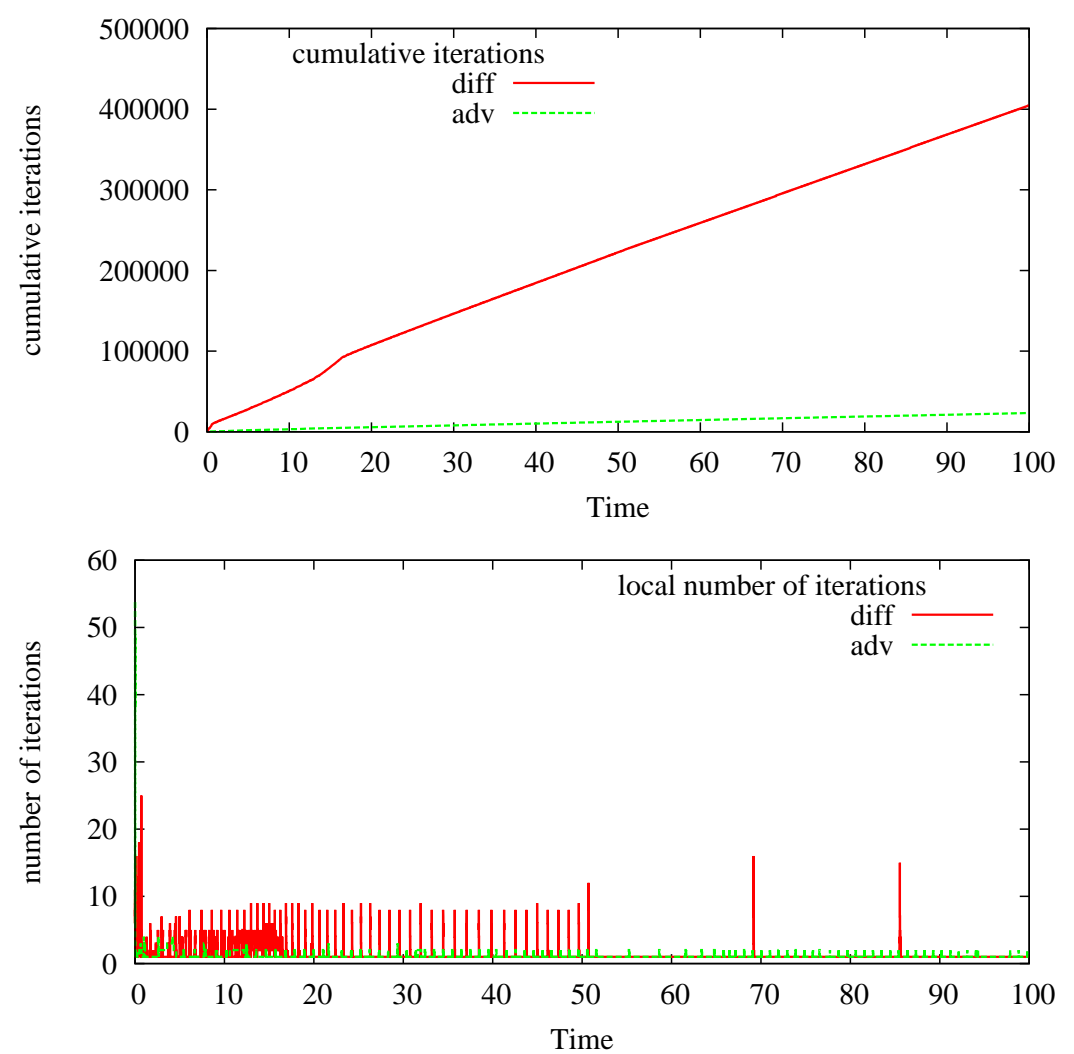

Fig. 15 Cumulative number of iterations (top) and number of coupling iterations (bottom) along the simulation for the dispersive and advective cases. The time step is free to evolve to accommodate for the local numerical stiffness within the Courant criterion $\left(f_{c}=10\right)$.

numerical stiffness is higher in the dispersive case than in the advective case, resulting in higher iteration outbursts (figure 15, bottom).

\section{Medium case}

The "medium" benchmark introduces two more derived species as well as a non-mobile species controlled by kinetics. The case defines different rates for dissociation and formation of the species, which is dealt with by HYTEC hence no modification of the code was required. No difficulties worthwhile noticing have been encountered. The oscillations as encountered in the easy case disappeared, due to a change in a thermodynamic constant and the role of the additional aqueous species. A detailed description of the medium case can be found in a separate report [16].

Figure 16 summarizes the CPU usage in function of the number of nodes for all simulations concerning the medium case. For a similar number of nodes, much more CPU is required for the dispersive case than for the advective case. CPU usage increases linearly on a log-log scale with the number of nodes, for all cases. CPU increase is stronger for the advective case: indeed, advective transport is much more restricted by the courant number 


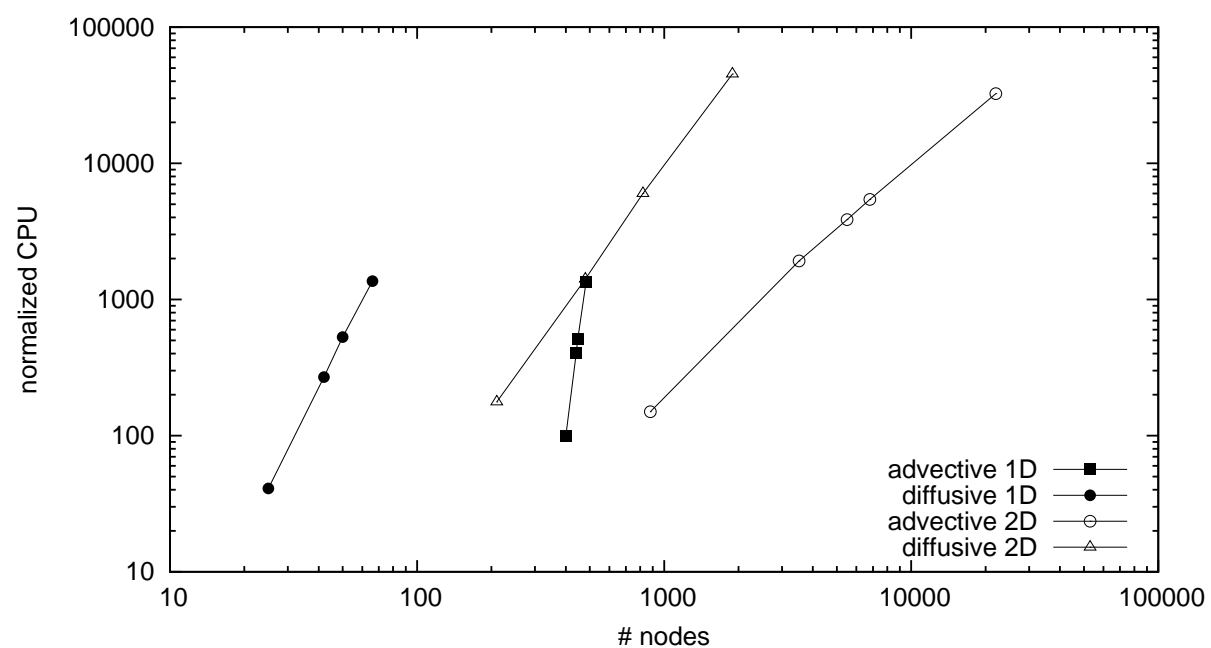

Fig. 16 Comparison of CPU usage for different grid refinements, for all simulations of the medium case.

criteria than dispersive transport, hence timesteps become rapidly very small for fine grids. Also, we can note that the slopes are different for advective and dispersive transport, which we believe is due to difference in courant-factor values (a HYTEC parameter which allows to overshoot the mathematical courant-number value). For similar courant-factor values, the slopes would become close to identical for all cases.

The precision of the HYTEC solution was validated both by convergence for finer grids simulations, and by comparisons with other teams results. However, one of the principle findings is that HYTEC provides a precise solution, even when using coarse grids. This is particularly true for the dispersive cases, where reactions are strongly coupled with transport. This was not unexpected, since the code is based on an iterative improvement of the coupling procedure. Without iterative improvement, coarse grids would allow larger timesteps and therefore become less precise. We therefore strongly recommend iterative improvement in all cases. This result is interesting for these computationally costly cases, where the HYTEC approach can provide a way to limit the number of nodes required for an accurate solution.

\section{Conclusion}

The easy test-case proposed by GdR MoMaS, though apparently quite simple with 4 basis components, 5 derived mobile species, and 3 fixed species, allows to bring forward several interesting numerical features. The most important one is the oscillation pattern observed for mobile species $\mathrm{C} 5$. The oscillations are a direct consequence of the discretization of space for a system at thermodynamic equilibrium, associated with a highly unstable species (large stoechiometric coefficients and large reaction constant). This effect can be reduced using finer grids (at the cost of increasing CPU), or by adding a small kinetic control, which might have a chemical sense in this context.

The reactive transport benchmark proposed by GdR MoMaS (test-case easy 1D) has been run by the current standard version of the reactive transport code HYTEC. The results provided in this paper follow the benchmark guidelines. Apart from the output imposed 
by the benchmark, additional information is provided to highlight the behavior of HYTEC specifically and the simulation results in particular.

All other benchmarks exercises have been completed using the standard version of HYTEC, without numerical difficulties worthwhile noticing. The oscillations found for the easy case and explained in detail in this paper where not observed for the medium test cases. Because the hard case requires the introduction of a non-physical decay option, not included in the standard release of HYTEC, we decided to leave this one out.

Our main conclusion is that HYTEC behaves stable and reliable, even for very stiff systems. CPU usage is reasonable (HYTEC can calculate the systems much faster using multi-processor computers, an option not used here for the obvious benchmark reasons). For the medium case study it has been shown that HYTEC provides a precise solution, even for coarse grids, provided the model obeys to the classical stability criteria. And we strongly believe that precision is what counts most in this context.

Acknowledgements HYTEC is developed within the framework of the Reactive Transport Consortium (PGT, Pôle Géochimie-Transport, http://pgt.geosciences.ensmp.fr). The authors acknowledge all partners of the PGT for financial support and helpful feedback which allows us to maintain and further develop our work on reactive transport modeling.

\section{References}

1. Bourgeat A., S. Bryant, J. Carrayrou, A. Dimier, C.J. van Duijn, M. Kern, P. Knabner (2006), Benchmark Reactive Transport, Technical Report GDR MOMAS.

2. Carrayrou J., V. Lagneau: The reactive transport benchmark proposed by GdR MoMaS (2007) Presentation and first results, In Eurotherm-81, Reactive Transport series, Albi.

3. Carrayrou J., J. Hoffmann, P. Knabner, S. Krutle, C. de Dieuleveult, J. Erhel, J. van der Lee, V. Lagneau, M. Kern, L. Amir, K.U. Mayer, K.T.B. MacQuarrie (submitted), A synthesis of the MoMaS reactive transport results, this issue.

4. Cochepin B., L. Trotignon, O. Bildstein, C.I. Steefel, V. Lagneau, J. van der lee (2008), Approaches to modelling coupled flow and reaction in a 2D cementation experiment, Advances in Water Resources, 31, 1540-1551.

5. De Simoni M., J. Carrera, X. Sánchez-Vila, A. Guadagnini (2005), A procedure for the solution of multicomponent reactive transport problems, Water Resour. Res, 41, 16.

6. De Windt L., R. Badreddine (2007), Modelling of long-term dynamic leaching tests applied to solidified/stabilised waste, Waste Management, 27, 1638-1647.

7. L. De Windt, A. Burnol, P. Montarnal, J. van der Lee (2003), Intercomparison of reactive transport models applied to UO2 oxidative dissolution and uranium migration, Journal of Contaminant Hydrology, 61, 303-312.

8. Lagneau V. (2003), R2D2 - Reactive Transport and Waterflow on an Odd Dimension 2 grid, notice technique et vérification, Technical Report Mines ParisTech LMH/RD/03/05.

9. Lagneau V. (2007), Benchmark reactive transport GdR MoMaS, HYTEC Ecole des Mines de Paris, Technical Report Mines ParisTech R060915VLAG.

10. Lagneau V., J. van der Lee (submitted), On the accuracy and efficiency of strongly coupled reactive transport models based on operator splitting: analytical solutions and test cases, Contaminant Hydrology.

11. Lasaga A.C. (1998) Kinetic theory of the Earth sciences, Princeton series in geochemistry. Princeton University press, $811 \mathrm{pp}$.

12. Lions J., J. van der Lee, V. Guren, P. Bataillard, A. Laboudigue (2007), Zinc and cadmium mobility in a 5-year-old dredged sediment deposit: Experiments and modelling, Journal of Soils and Sediments, 7, 207-215.

13. Srinivasana V., T.P. Clement (2008), Analytical solutions for sequentially coupled one-dimensional reactive transport problems Part I: Mathematical derivations

14. Steefel C.I., , D.J. DePaoloa, Lichtner P.C. (2005), Reactive transport modeling: An essential tool and a new research approach for the Earth sciences, Earth and Planetary Science Letters, 240, 539-558.

15. Sun Y., L. Glascoe (2006), Modeling biodegradation and reactive transport: Analytical and numerical models, ACS Symposium Series, 940, 153-174. 
16. van der Lee J., V. Lagneau (2008), GDR MOMAS - benchmark reactive transport: HYTEC results of the medium and hard benchmark cases, Technical Report Mines ParisTech R080403JVDL.

17. van der Lee J. (1998), Thermodynamic and mathematical concepts of CHESS, Technical Report Mines ParisTech LHM/RD/98/39.

18. van der Lee J., L. De Windt, V. Lagneau, P. Goblet (2002), Presentation and application of the reactive transport code HYTEC, Computational methods in water resources, 1, 599-606.

19. van der Lee J., L. De Windt, V. Lagneau, P. Goblet (2003), Module-oriented modeling of reactive transport with HYTEC, Computers \& Geosciences, 29, 265-275.

20. Yeh G.T., V.S. Tripathi (1989), A critical evaluation of recent developments in hydrogeochemical transport models of reactive multi-chemical components, Water Resources Research, 25 93-108.

21. Zhao C., B.E. Hobbs, P. Hornby, A. Ord, S. Peng, L. Liu (2007), Theoretical and numerical analyses of chemical-dissolution front instability in fluid-saturated porous rocks, International Journal for Numerical and Analytical Methods in Geomechanics, 32, 1107-1130. 